\title{
TARSKI SEMIGROUPS
}

\author{
ERIK ELLENTUCK
}

\begin{abstract}
A Tarski semigroup is an algebraic system which mirrors a fragment of the additive theory of cardinal numbers. Here we show that any two such systems have the same universal theory. We also give a simple arithmetical necessary and sufficient condition for a universal sentence to hold in a Tarski semigroup.
\end{abstract}

1. Introduction. A system $(S,+, 0)$, where + is a binary operation on $S$ and 0 is a distinguished element of $S$, is a Tarski semigroup (TSG) if it satisfies the universal closures of (1)-(6) below.

(1) $x+(y+z)=(x+y)+z$,

(2) $x+0=x=0+x$,

(3) $x+y=y+x$,

(4) $x+y=0 \rightarrow x=0$,

(5) $x+z=y+z \rightarrow x=y$,

(6) $n x=n y \rightarrow x=y$,

where $0<n<\omega$ is a positive integer and $n x$ is $x$ summed with itself $n$ times. Let $S$ be a TSG. $S$ is nontrivial if it contains at least two elements. For $x, y \in S$ define $x \leqslant y$ if there is a $z \in S$ such that $x+z=y$. Then under this definition $S$ is a partially ordered semigroup. The only interesting case is antisymmetry. If $x+a=y$ and $y+b=x$ then

$$
x+(a+b)=(x+a)+b=y+b=x=x+0 .
$$

Then $a+b=0$ by (5), $a=0$ by (4), and $x=y$ by (2). Familiar examples of TSG's are (i) the nonnegative integers under addition $(\omega,+, 0)$, (ii) the positive integers under multiplication $(\omega-\{0\}, \cdot, 1)$, (iii) the isols under addition $(\Lambda,+, 0)$, (iv) the positive isols under multiplication $(\Lambda-\{0\}, \cdot 1)$, (v) the Dedekind cardinals under addition $(\Delta,+, 0)$, and (vi) the positive Dedekind cardinals under multiplication $(\Delta-\{0\}, \cdot, 1)$.

Let $L$ be a first order language with equality containing a binary function symbol + and a 0 -ary function symbol $0 . L$ has its usual interpretation in any TSG. When $(S,+, 0)$ is specified we always confuse + with + , and 0 with 0 . Formally, sentences of $L$ will be written in prenex conjunctive normal form, although in application all sorts of abbreviations will be used. If $\varphi$ is a sentence then a Horn reduct of $\varphi$ is any one of the sentences obtained from $\varphi$

Received by the editors October 29, 1976.

AMS (MOS) subject classifications (1970). Primary 06A50, 02F40.

Key words and phrases. The Farkas Lemma.

- American Mathematical Society 1977 
by striking out from each conjunct of its matrix all but at most one unnegated atomic formula. $\varphi$ is a Horn sentence if it is one of its own Horn reducts. $\varphi$ is universal if its prefix contains only universal quantifiers. The universal theory of $S$ is the set of all universal sentences which hold in $S$.

THEOREM. Any two nontrivial TSG's have the same universal theory. In fact, if $\varphi$ is a universal sentence of $L$ and $S$ is a nontrivial TSG, then $\varphi$ holds in $S$ if and only if some Horn reduct of $\varphi$ holds in $(\omega,+, 0)$.

The necessary and sufficient condition given in our theorem has long been known to hold for examples (iii)-(vi) above. In each case they were obtained by detailed set theoretic reasoning. What is interesting here is how they can also be obtained from such a simple basis as the axioms for a TSG. Suppose that $\varphi$ is a universal sentence (of some language) that holds in $\omega$ but having no Horn reduct holding in $\omega$. To show that $\varphi$ fails in the isols usually requires some infinite pathological isol. Curiously, such an object is not required for our theorem. No doubt this is due to the simplicity of $L$.

We would like to thank D. Slater for his criticism of a weaker version of our theorem.

2. Proof. Our proof depends on a remarkable result in the theory of linear inequalities which is due to J. Farkas (cf. [1]). Let $V_{n}$ consist of the set of all $n$-tuples of rational numbers considered as a vector space over the rationals. Use - for the inner product. Then we have the

FARKAS Lemma. Let $b, a_{i}, i<m$, be vectors in $V_{n}$. If for all $x \in V_{n}, b \cdot x \geqslant$ 0 whenever $a_{i} \cdot x \geqslant 0$ for each $i<m$, then there exist nonnegative rationals $r_{i}, i<m$, such that $b=\Sigma_{i<m} r_{i} a_{i}$.

LEMMA 1. If $\varphi$ is a universal Horn sentence of $L$ and $\varphi$ holds in $\omega$ then $\varphi$ holds in every TSG.

Proof. Let $\varphi$ be a universal Horn sentence holding in $\omega$. A universal sentence holds in a system if and only if the universal closure of each conjunct of its matrix holds in that system. Thus we may assume that the matrix of $\varphi$ is a single conjunct, i.e., it is a disjunction of atomic formulas and their negations, at most one atomic formula being unnegated. If all are negated then $\varphi$ fails in every TSG because a system of homogeneous equations can always be satisfied in a TSG if we assign 0 to each variable. Thus we may assume that the matrix of $\varphi$ can be abbreviated as an implication. Suppose that $\left(\forall u_{0}\right) \ldots\left(\forall u_{n-1}\right)$ is the prefix of $\varphi$. Then each term of $\varphi$ is equivalent (mod the TSG axioms) to one of the form $\sum_{i<n} a_{i} u_{i}$ where $0<a_{i}<\omega$ for $i<n$. Thus we can express $\varphi$ in vector-matrix notation as

$$
(\forall u)\left(A_{0} u=A_{1} u \rightarrow B_{0} u=B_{1} u\right)
$$

where $u=\left(u_{0}, \ldots, u_{n-1}\right)$ is a column vector of variables and $A_{i}, B_{i}$ are matrices all of whose entries are nonnegative integers. Note that "matrix" is used in two ways. We have tried to keep them contextually distinct. (7) is 
somewhat generous because the $B_{i}$ need only have a single row. By adding zeros if necessary we may take the $A_{i}, B_{i}$ as $m \times n$ matrices.

Let $A=A_{0}-A_{1}, B=B_{0}-B_{1}$ and $D=$ the $n \times n$ diagonal matrix all of whose diagonal elements are equal to $1 .\left(\omega^{*},+, 0\right)$ is the group of rational integers. Since (7) holds in $(\omega,+, 0)$,

$$
(\forall u)(A u=0 \wedge D u \geqslant 0 \rightarrow B u=0)
$$

holds in $\left(\omega^{*},+, 0\right)$. Let

$$
\tilde{A}=\left(\begin{array}{c}
A \\
-A \\
D
\end{array}\right)
$$

Then

$$
\begin{gathered}
(\forall u)(\tilde{A} u \geq 0 \rightarrow B u \geqslant 0), \\
(\forall u)(\tilde{A} u \geqslant 0 \rightarrow-B u \geqslant 0) ;
\end{gathered}
$$

both hold in $\left(\omega^{*},+, 0\right)$. Let $\left(\omega^{\#},+, 0\right)$ be the group of rational numbers. By removing and then replacing a positive integral common denominator it follows that (9) and (10) both hold in $\left(\omega^{\#},+, 0\right)$. We can now apply the Farkas Lemma, say, first to (9). Each row of $B$ is a nonnegative rational linear combination of the rows of $\tilde{A}$. By removing a positive integral common denominator $s$ we get a matrix $P$ whose entries are all nonnegative integers such that

$$
s B=P \tilde{A} .
$$

The same process applied to (10) gives us a positive integer $t$ and a matrix $Q$ whose entries are all nonnegative integers such that

$$
-t B=Q \tilde{A} \text {. }
$$

Let $P=\left(P_{0} P_{1} P_{2}\right)$ where $P_{0}$ and $P_{1}$ both have $m$ columns and $P_{2}$ has $n$ columns. Then $P \tilde{A}=P_{0} A-P_{1} A+P_{2} D$. Replacing $A$ by $A_{0}-A_{1}$ and $B$ by $B_{0}-B_{1},(11)$ after transposing terms, gives us

$$
s B_{0}+P_{0} A_{1}+P_{1} A_{0}=s B_{1}+P_{0} A_{0}+P_{1} A_{1}+P_{2} D .
$$

By doing the same thing to (12) we get

$$
t B_{1}+Q_{0} A_{1}+Q_{1} A_{0}=t B_{0}+Q_{0} A_{0}+Q_{1} A_{1}+Q_{2} D .
$$

Now suppose that $S$ is any TSG and $x$ is an $n$-ary column vector whose entries belong to $S$ such that $A_{0} x=A_{1} x$. Apply (13) to $x$ and use (5) to cancel the equal terms $P_{1} A_{0} x=P_{1} A_{1} x$ and $P_{0} A_{1} x=P_{0} A_{0} x$ to get $s B_{0} x=$ $s B_{1} x+P_{2} D x$, i.e.,

$$
s B_{1} x \leqslant s B_{0} x
$$

Using (14) instead we obtain

$$
t B_{0} x \leqslant t B_{1} x
$$

Multiplying (15) by $t$ and (16) by $s$, we get $s t B_{0} x<s t B_{1} x<s t B_{0} x$. Then st $B_{0} x=s t B_{1} x$ since $S$ is a partially ordered semigroup, and finally $B_{0} x=$ $B_{1} x$ by (6). Q.E.D. 
LEMMA 2. If $\varphi$ is a universal sentence of $L$ and $\varphi$ holds in $(\omega,+, 0)$, then some Horn reduct of $\varphi$ will also hold in $(\omega,+, 0)$.

Proof. Let $\varphi$ be a universal sentence holding in $(\omega,+, 0)$. Then just as in the proof of Lemma 1 we can express $\varphi$ as

$$
(\forall u)\left(A_{0} u=A_{1} u \rightarrow \underset{i<k}{\bigvee} B_{i 0} u=B_{i 1} u\right)
$$

where $u$ is an $n$-tuple of variables. Our proof is by induction on $n$. We leave the basis step to the reader. Let $A=A_{0}-A_{1}, B_{i}=B_{i 0}-B_{i 1}$, and $D=$ the $n \times n$ diagonal matrix whose diagonal elements are all equal to 1 . Since (17) holds in $(\omega,+, 0)$,

$$
(\forall u)\left(A u=0 \wedge D u \geqslant 0 \rightarrow \bigvee_{i<k} B_{i} u=0\right)
$$

holds in $\left(\omega^{\#},+, 0\right)$. Let $A^{0}, B_{i}^{0}$, and $P$ be, respectively, the set of vectors $x \in V_{n}$ which satisfy $A x=0, B_{i} x=0$, and $D x \geqslant 0$. From (18) we have $A^{0} \cap P \subseteq \cup_{i<k} B_{i}^{0}$ and, hence,

$$
A^{0} \cap P=\bigcup_{i<k}\left(A^{0} \cap B_{i}^{0} \cap P\right) .
$$

The dimension of $A^{0} \cap P$ is the maximal number of linearly independent vectors which it contains. Suppose that it is $s$ (note that $s$ may be strictly less than the dimension of $A^{0}$ ). If the dimension of some $A^{0} \cap B_{j}^{0} \cap P$ is also equal to $s$ then $A^{0} \cap P=A^{0} \cap B_{j}^{0} \cap P$ and, hence, $(\forall u)\left(A_{0} u=A_{1} u \rightarrow B_{j 0} u\right.$ $\left.=B_{j 1} u\right)$ holds in $(\omega,+, 0)$. Thus we may suppose that every $A^{0} \cap B_{i}^{0} \cap P$ has dimension strictly less than $s$. Suppose that $A^{0} \cap P$ contains no vector all of whose components are positive. Then there is a $j<n$ such that every vector in $A^{0} \cap P$ vanishes at its $j$ th component. For, otherwise, choose $x_{i} \in A^{0} \cap P$ for $i<n$ such that $x_{i}$ is positive at its $i$ th component. Then $\sum_{i<n} x_{i} \in A^{0} \cap P$ and is positive at all components. If every vector in $A^{0} \cap P$ vanishes at its $j$ th component, then the variable $u_{j}$ may be eliminated in (17) and our lemma follows by the induction hypothesis. Thus we may assume that $A^{0} \cap P$ contains a vector $x$ which is positive at all its components.

Let $\rho$ be the distance function in $V_{n}$, and for rational $\varepsilon>0$ let $S(x, \varepsilon)=$ $\left\{y \in V_{n} \mid \rho(x, y)<\varepsilon\right\}$. Choose $\varepsilon$ so small that every $y \in S(x, \varepsilon)$ is positive at all its components. We claim that $A^{0} \cap P \cap S(x, \varepsilon)$ contains $s$ linearly independent vectors. Let $a_{i} \in A^{0} \cap P$ for $i<s$ be linearly independent vectors and $y_{i}(t)=(1-t) x+t a_{i}$ for rational $0 \leqslant t \leqslant 1$. Let $M(t)$ be the $s \times n$ matrix whose rows are $y_{i}(t)$. Since the rows of $M(1)$ are linearly independent, $M(1)$ contains an $s \times s$ nonvanishing determinant. Let $d(t)$ be the corresponding determinant in $M(t)$. Thus $d(t)$ is a nontrivial polynomial in $t$ because $d(1) \neq 0$. Clearly $y_{i}(t) \in A^{0} \cap P$ for $i<s$ and rational $0 \leqslant t<$ 1. Then for some sufficiently small positive rational $t^{\prime}, \rho\left(y_{i}\left(t^{\prime}\right), x\right)<\varepsilon$ for $i<s$, and $d\left(t^{\prime}\right) \neq 0$; the latter insuring that the $y_{i}\left(t^{\prime}\right)$ are linearly independent.

Now $A^{0} \cap B_{0}^{0} \cap P$ has dimension smaller than $s$ and hence we can find an 
element $x_{0} \in\left(A^{0} \cap S(x, \varepsilon)\right)-B_{0}^{0}$. Since $B_{0}^{0}$ is a closed set, we can then find an $\varepsilon_{0}$ such that $S\left(x_{0}, \varepsilon_{0}\right) \subseteq S(x, \varepsilon)$ and $S\left(x_{0}, \varepsilon_{0}\right)$ is disjoint from $B_{0}^{0}$. Continuing in this way we get a decreasing sequence of spheres $S\left(x_{i}, \varepsilon_{i}\right)$, $i<k$, such that $x_{i} \in A^{0} \cap P$ and $S\left(x_{i}, \varepsilon_{i}\right)$ is disjoint from $B_{i}^{0}$. But then the existence of $x_{k-1}$ contradicts (19). Q.E.D.

Proof of Theorem. Suppose that $\varphi$ is a universal sentence holding in $(\omega,+, 0)$ and $S$ is a nontrivial TSG. By Lemma $2, \varphi$ has a Horn reduct $\psi$ holding in $(\omega,+, 0)$, and by Lemma $1, \psi$ also holds in $S$. That $\varphi$ holds in $S$ follows by predicate calculus. Conversely, if $\varphi$ holds in $S$ choose a nonzero $x \in S$. Then $S_{0}=\{n x \mid n<\omega\} \subseteq S$ is isomorphic to $(\omega,+, 0)$. Since $\varphi$ is universal, it holds in $S_{0}$ and hence in $(\omega,+, 0)$.

\section{REFERENCES}

1. J. Farkas, Über die Theorie der einfachen Ungleichungen, J. Reine Angew. Math. 124 (1902), $1-27$.

Department of Mathematics, Rutgers University, New Brunswick, New Jersey 08903

Current address: Department of Mathematics, University of California, Berkeley, California 94720 